LA W RENCE LIVERMORE N A T IO N A L LABORATORY

Tunable Diode Laser Absorption Spectroscopy Using a Multi-Pass White Cell for O2 Detection

M. Raval, M. Bora, J. McCarrick, T. Bond

August 14, 2012 
This document was prepared as an account of work sponsored by an agency of the United States government. Neither the United States government nor Lawrence Livermore National Security, LLC, nor any of their employees makes any warranty, expressed or implied, or assumes any legal liability or responsibility for the accuracy, completeness, or usefulness of any information, apparatus, product, or process disclosed, or represents that its use would not infringe privately owned rights. Reference herein to any specific commercial product, process, or service by trade name, trademark, manufacturer, or otherwise does not necessarily constitute or imply its endorsement, recommendation, or favoring by the United States government or Lawrence Livermore National Security, LLC. The views and opinions of authors expressed herein do not necessarily state or reflect those of the United States government or Lawrence Livermore National Security, LLC, and shall not be used for advertising or product endorsement purposes.

This work performed under the auspices of the U.S. Department of Energy by Lawrence Livermore National Laboratory under Contract DE-AC52-07NA27344. 


\title{
Tunable Diode Laser Absorption Spectroscopy Using a Multi-Pass White Cell for $\mathrm{O}_{2}$ Detection
}

\author{
Manan Raval ${ }^{1,2}$, Mihail Bora ${ }^{1}$, Jim McCarrick ${ }^{1}$, Tiziana Bond ${ }^{1, *}$ \\ ${ }^{1}$ Lawrence Livermore National Laboratory, Livermore, CA 94550; ${ }^{2}$ University of Illinois at \\ Urbana-Champaign, Urbana, IL 61820; *bond7@1lnl.gov
}

\begin{abstract}
Two forms of Tunable Diode Laser Absorption Spectroscopy (TDLAS), direct absorbance spectroscopy and wavelength modulation spectroscopy (WMS), for $\mathrm{O}_{2}$ detection were carried out using a tunable 763-nm vertical cavity surface emitting laser (VCSEL) in a multi-pass White cell with an optical path length of $2.6 \mathrm{~m}$. The cell was fitted with a gas flow mechanism for concentration studies. The output wavelength of the tunable VCSEL was increased by ramping the drive current from 0-5 mA while temperature controlling the device to $20{ }^{\circ} \mathrm{C}$. The output wavelength of the VCSEL was mapped using a spectrum analyzer. The wavelength could be tuned approximately $3 \mathrm{~nm}$ by ramping the current. Direct absorbance spectroscopy involves reading the transmission of the laser at the output of the White cell. A photodetector is placed at the end of the optical path for this purpose. Absorption lines appear as dips in the transmission. WMS involves modulating the drive current and extracting the second harmonic of the photodetector output. This method is more sensitive due to the lack of a background intensity and lower noise at higher frequencies. The lower limit of detection observed in individual measurements for both methods was determined. WMS yielded a limit of detection of $200 \mathrm{ppm}$, while direct absorbance spectroscopy yielded a limit of detection of approximately $2000 \mathrm{ppm}$. The collected data were fitted with a Voigt profile to extract values for concentration and spectral linewidth. The fitted parameters for the concentration were within $15 \%$ of the expected value, which was calculated for the ambient testing conditions using the ideal gas law. On the other hand the fitted parameters for the linewidth were approximately twice the expected value, meaning that there may be additional broadening effects that may not have been taken into account.
\end{abstract}

\section{Introduction}

The detection of gases and concentration measurements are necessary for various industrial applications as well as environmental analysis. Several gases, such as oxygen and carbon dioxide, exhibit strong absorption of light in the infrared range. Thus, absorption spectroscopy serves as an effective means of gas detection.

Direct absorbance spectroscopy and wavelength modulation spectroscopy were carried out and analyzed as a continuation of the work carried out previously [1]. In both cases, a 763$\mathrm{nm}$ VCSEL was used to sense oxygen. The same mechanism applies for sensing other gases as well, given that a laser with an output wavelength corresponding to strong absorption lines of the desired gas is chosen. In either case the output wavelength of the VCSEL was tuned by changing 
the drive current, which changes the laser temperature. This change in current alters the polarization of the VCSEL along a crystal axis, thereby changing the output wavelength [2]. For the 763-nm VCSEL, which is driven with up to $5 \mathrm{~mA}$ of current, the range of the output wavelength is about $3 \mathrm{~nm}$.

The absorption lines seen in measurements must be compared with the spectral lines defined in the HITRAN database for the measured gas species [3]. The measured spectral lines can then be fitted using a Voigt profile, which is the convolution of the Lorentzian profile to account for natural and pressure broadening and the Gaussian profile to account for thermal Doppler broadening. Pressure broadening dominates at high pressures while Doppler broadening dominates at low pressures or high temperatures. At moderate temperatures and pressures around $1 \mathrm{~atm}$, both broadening mechanisms must be considered and a Voigt profile becomes necessary $[3,4]$. The experiment was carried out at approximately standard temperature and pressure, at which all of the previously mentioned broadening mechanisms are factors. The concentration of the gas is determined as a parameter through the fitting.

\section{Method}

Absorption lines for $\mathrm{O}_{2}$ in the visible region near $760 \mathrm{~nm}$ are strong and very narrow, allowing them to be isolated and studied individually. These absorption lines arise from the rotational and vibrational excitation of gas molecules at discrete resonant wavelengths. These spectral lines undergo wavelength broadening due to intermolecular collisions and thermally induced velocities [4]. VCSELs provide the bright and highly monochromatic light necessary to resolve these spectral lines [4]. The objective of any form of TDLAS is to tune the output wavelength of the laser in order to fully scan an absorption line or a series of absorption lines. In VCSELs, this tuning is done by either adjusting the temperature or ramping the current of the VCSEL [4]. In this experiment, the temperature of the VCSEL was maintained at $20{ }^{\circ} \mathrm{C}$ in order to eliminate any wavelength variance due to temperature. The current was then ramped up from 0 to $5 \mathrm{~mA}$, which caused the output wavelength to increase in a sublinear fashion

In both detection methods, the setup was enclosed in a type of multi-pass absorption cell known as a White cell [5]. The White cell, acquired from the Fraunhofer Institute for Physical

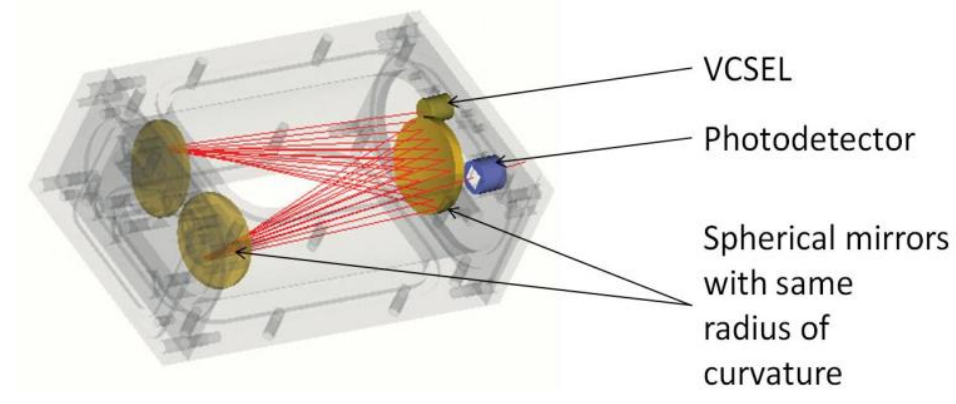

Fig. 1. Multi-pass White Cell

Measurement Techniques in

Freiburg, Germany, was fitted with a gas inlet and a drain to allow for concentration studies of oxygen, which was introduced into the cell with nitrogen. The total flow of gas through the cell at any given point in an experiment was $5000 \mathrm{sccm}$. The total optical path length of the White cell was $2.6 \mathrm{~m}$ with a $10 \mathrm{~cm}$ cavity length. A schematic of the White cell is shown in Fig. 1. For both methods, a concentration sweep of $\mathrm{O}_{2}$ from 0 to $100 \%$ in $2 \%$ increments as well as a low concentration sweep from 0 to $1 \%$ in $0.02 \%$ increments was carried out. A ULM Photonics 763 - 
$\mathrm{nm}$ VCSEL was placed on the input end of the White cell and a photodetector was placed at the output.

Two photodetectors - a Hamamatsu K3413-08 two-color detector with a built in Si-based detector and a Thorlabs PDA100A Si-based detector - were tested. The Hamamatsu detector exhibited an etalon effect at the viewing window, as depicted in Fig. 3a. This led to a nonconstant background from measurement to measurement. The Thorlabs detector, on the other hand, exhibited no such effect and maintained a constant background throughout all measurements.

In direct absorbance spectroscopy, the drive current of the VCSEL was ramped up from 0 to $5 \mathrm{~mA}$ and the output was read at the detector so as to produce an LI curve. Fig. 2 shows a schematic of the direct absorbance setup. As shown in Fig. 3, $\mathrm{O}_{2}$ absorption lines appear as expected at the output.

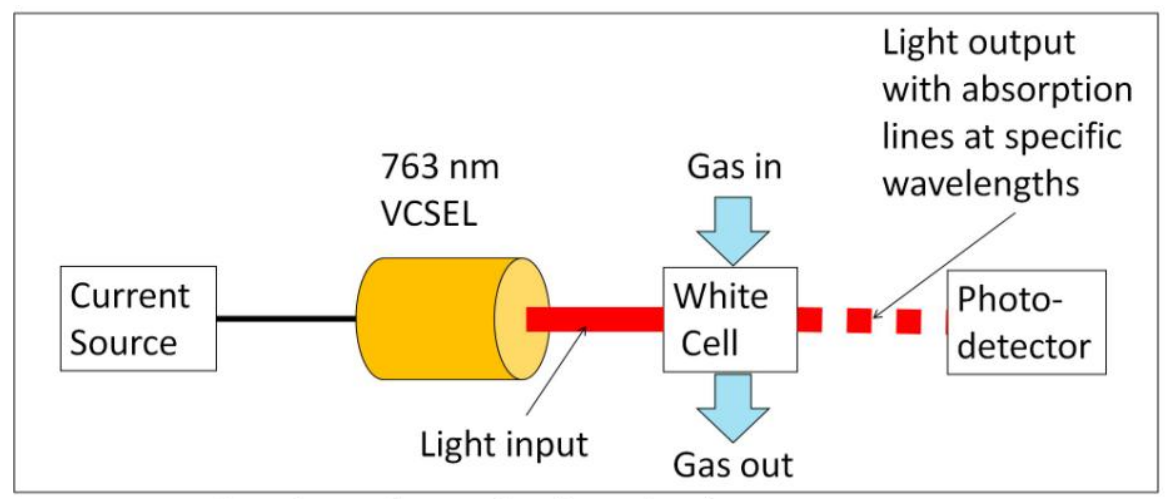

Fig. 2. Experimental setup for direct absorbance spectroscopy

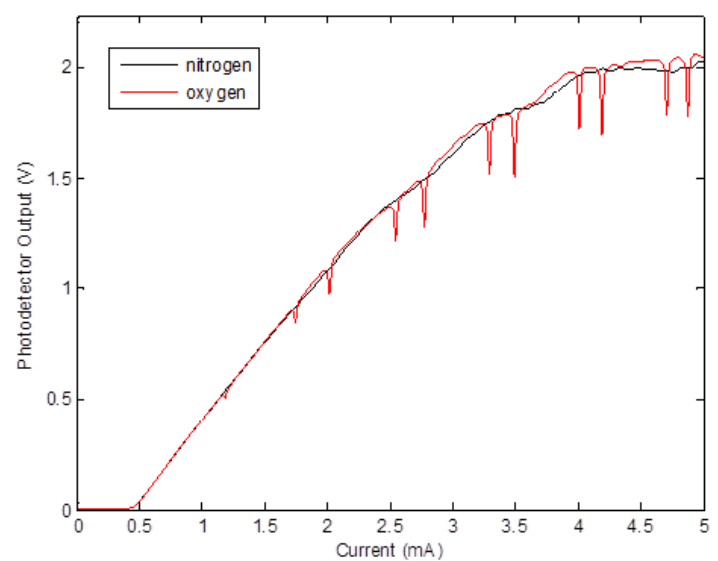

Fig. 3a. LI curve with Hamamatsu Detector

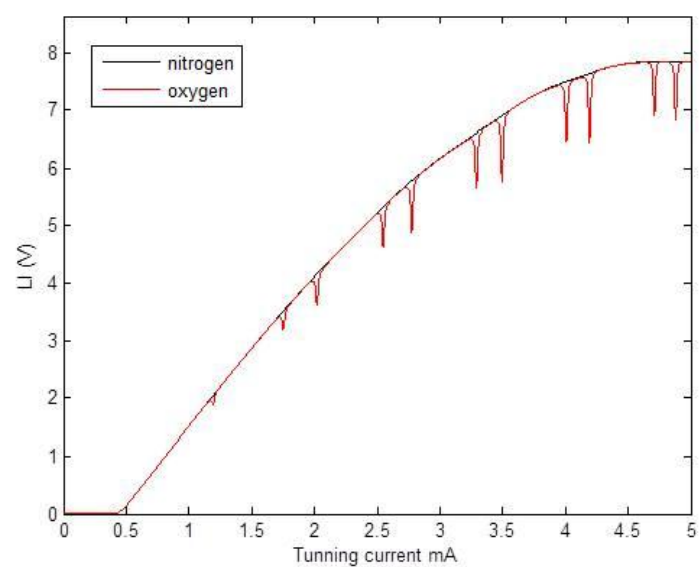

Fig. 3b. LI curve with Thorlabs Detector

In wavelength modulation spectroscopy, the drive current of the VCSEL was modulated with a frequency of $5 \mathrm{kHz}$ and amplitude of $35 \mu \mathrm{A}$. These optimum values for the frequency and amplitude were determined experimentally. The output seen at the detector was then directed to a lock-in amplifier which multiplies the signal by a $2 \omega$, or $10 \mathrm{kHz}$, sine wave in order to extract the second harmonic of the output. Fig. 4 shows a schematic of the wavelength modulation spectroscopy setup. The second harmonic, as shown in Fig. 5, was chosen because of its high 
sensitivity to low concentrations, its relatively simple shape, its lack of a background, and lower noise at higher frequencies.

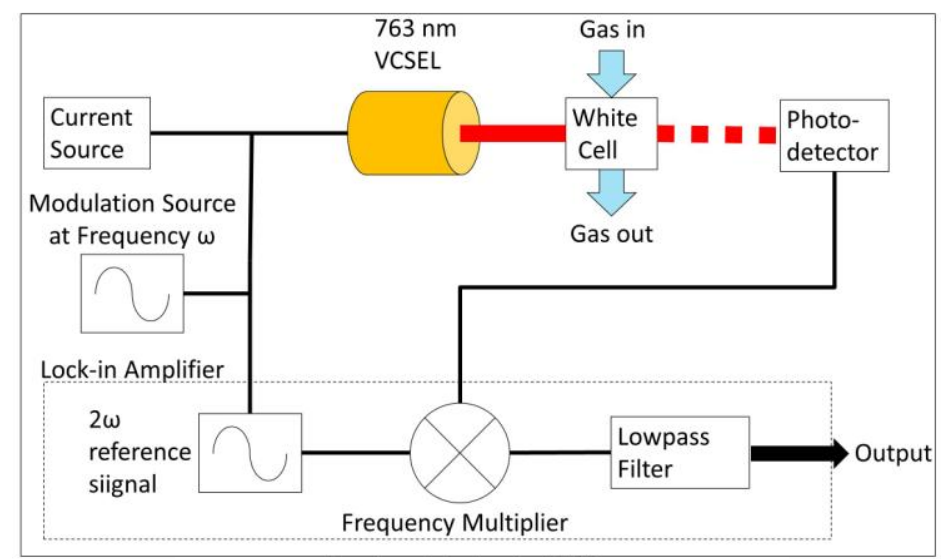

Fig 4. Experimental Setup for WMS

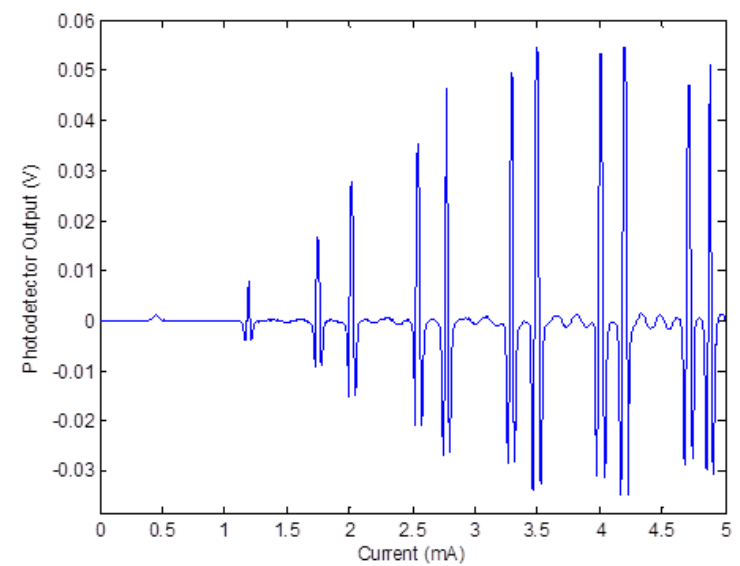

Fig. 5a. WMS with Hamamatsu Detector

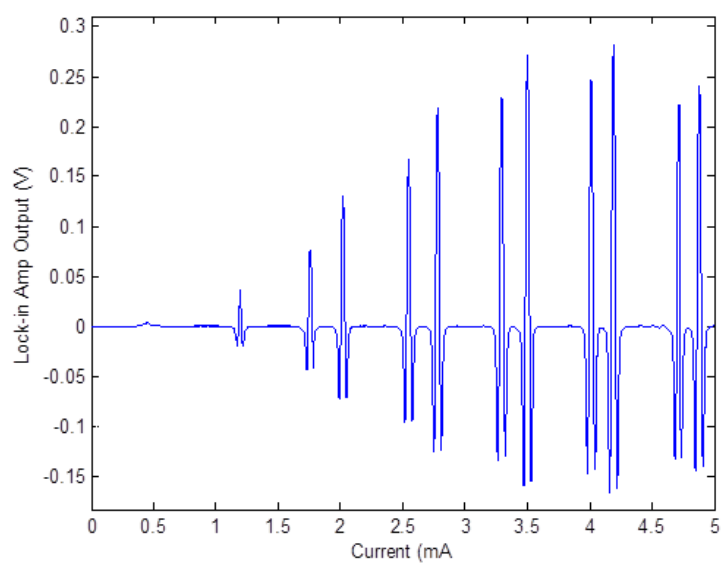

Fig. 5b. WMS with Thorlabs Detector

Because of its higher sensitivity, WMS is the preferred method. This form of spectroscopy is sensitive to $0.02 \% \mathrm{O} 2$ or $200 \mathrm{ppm}$. For the purpose of limit-of-detection studies, the LI curve is represented as absorbance $A$ defined by the expression below:

$$
A=\ln \left(\frac{I}{I_{0}}\right)
$$

where $I$ is the transmitted intensity and $I_{0}$ is the background intensity. Fig. 6a shows the amplitude of the absorbance for the highest peak for a range of concentration from $0-0.28 \%$. A peak can be distinguished as low as $0.2 \%$, or $2000 \mathrm{ppm}$. Fig. 6b shows the amplitude of the WMS data for one of the peaks for a range of concentration from $0-0.2 \%$. A peak is discernable as low as $0.02 \%$ or 200 ppm. 


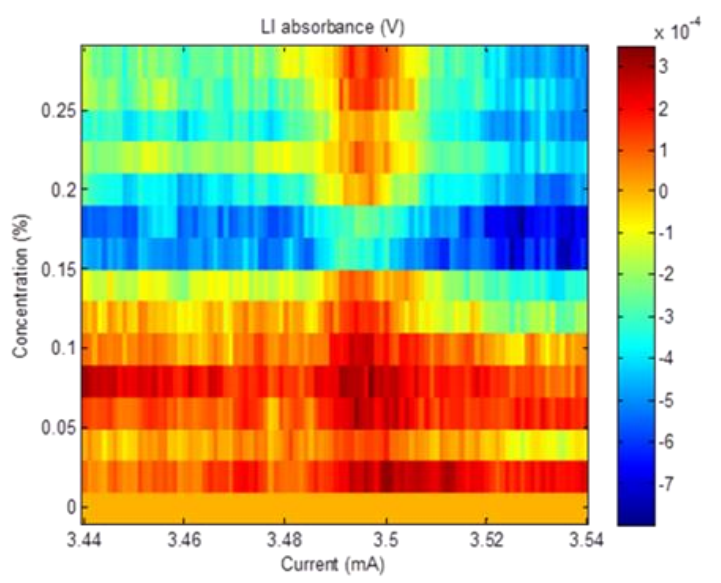

Fig 6a. Absorbance amplitude vs. concentration

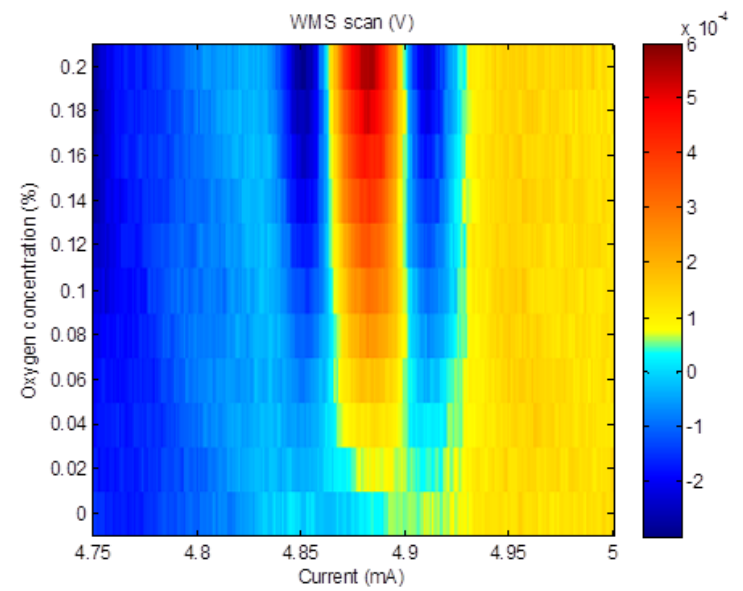

Fig 6b. WMS amplitude vs. concentration

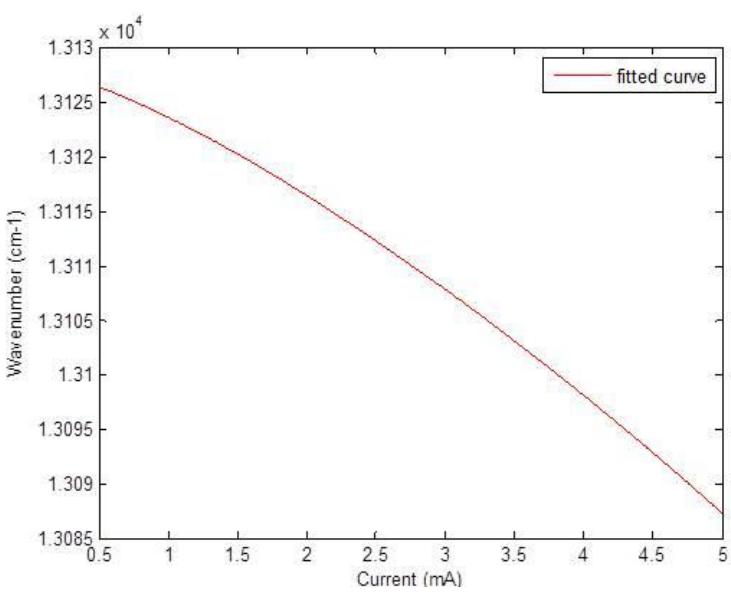

Fig. 7. Current-wavenumber relationship

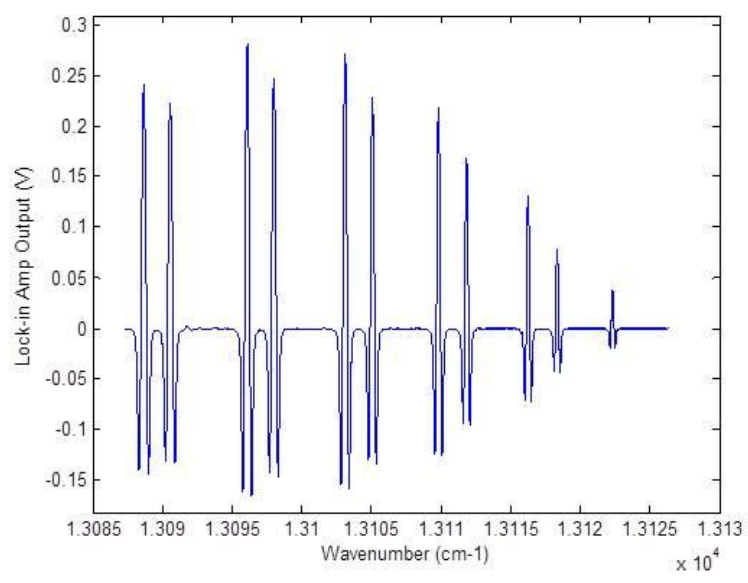

Fig. 8b. WMS with Thorlabs detector

Fig. 8a. LI curve with Thorlabs detector 
Fig. 9 depicts a comparison of the collected data to HITRAN values with respect to central frequencies. As can be seen in the figure, there is a significant offset between the measured central frequencies. This is most likely because of a lack of accuracy in the calibration of the spectrum analyzer itself due to the fact that the instrument does not use a reference laser. The current-wavenumber calibration will need to be carried out again in the future with wellcalibrated equipment.

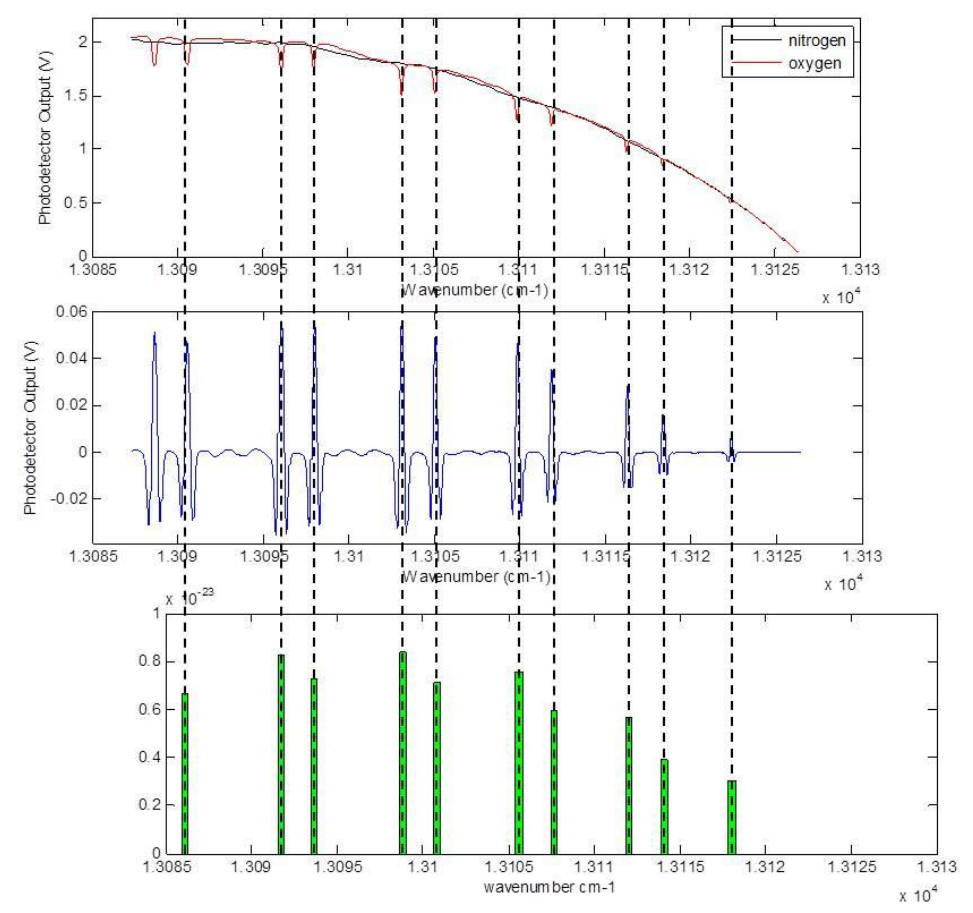

Fig. 9. Central frequency comparison with HITRAN

\section{Data Analysis}

When carrying out data analysis, it is necessary to express the data in terms of wavenumber. This enables comparisons with the values for spectral width, central wavelength, and spectral line strength provided by HITRAN. The current is calibrated to frequency in wavenumber by first finding the relation between current and wavelength using a spectrum analyzer. The wavenumber in $\mathrm{cm}^{-1}$ is one over the wavelength in $\mathrm{cm}$.

A Voigt profile, which is a convolution of the Lorentzian and Doppler profiles, can be estimated with an accuracy of 1-2\% using the expression shown below [7]. The expression for the profile divided by the value of the profile at the central frequency is given by: 


$$
\begin{aligned}
\frac{g_{V}(\sigma)}{I g_{V_{\max }}}=\{[1 & \left.\left.-\frac{w_{l}}{w_{v}}\right] \exp \left[-2.772\left(\frac{D N(\sigma)}{w_{v}}\right)^{2}\right]\right\}+\left\{\frac{\left(\frac{w_{l}}{w_{v}}\right)}{1+4\left(\frac{D N(\sigma)}{w_{v}}\right)^{2}}\right\} \\
& +0.016\left(1-\frac{w_{l}}{w_{v}}\right)\left(\frac{w_{l}}{w_{v}}\right)\left\{\exp \left[-0.4\left(\frac{D N(\sigma)}{w_{v}}\right)^{2.25}\right]-\frac{10}{10+\left(\frac{D N(\sigma)}{w_{v}}\right)^{2.25}}\right\}
\end{aligned}
$$

where $w_{l}$ and $w_{d}$ are the FWHM linewidths of the Lorentzian and Doppler profiles in $\mathrm{cm}^{-1}$ and $w_{v}$ is the Voigt linewidth in $\mathrm{cm}^{-1}$ defined as:

$$
w_{v}=0.5346 \cdot w_{l}+\sqrt{0.2166 \cdot w_{l}^{2}+w_{d}^{2}}
$$

The value of the central frequency of the Voigt profile is given in $\mathrm{cm}$ by:

$$
I g_{V_{\max }}=\frac{1}{w_{v}\left[1.065+0.447\left(\frac{w_{l}}{w_{v}}\right)+0.058\left(\frac{w_{l}}{w_{v}}\right)^{2}\right]}
$$

$D N$ is difference in frequency from the central frequency in $\mathrm{cm}^{-1}$ defined as

$$
D N(\sigma)=\sigma-\sigma_{0}
$$

where $\sigma$ is the frequency and $\sigma_{0}$ is the central frequency in wavenumber of a given spectral line [3].

The photodetector reads the transmission, which is expressed with respect to the baseline $I_{0}$ as:

$$
T=I_{0} \exp \left(-c d S g_{V}(\sigma)\right)
$$

where $c$ is concentration in molecules $/ \mathrm{cm}^{3}, d$ is the total optical path length in $\mathrm{cm}$, and $S$ is the line strength in $\mathrm{cm} /$ molecule. As is seen in Fig. 1b, the baseline of the LI curve is constant regardless of the concentration. This allows for the baseline to be determined by a polynomial fit of the $0 \% \mathrm{O}_{2}$ curve. Each spectral line is then fit individually to the expression shown below:

$$
T=\left(p_{4} \sigma^{4}+p_{3} \sigma^{3}+p_{2} \sigma^{2}+p_{1} \sigma+p_{0}\right) \exp \left(-c d S g_{V}(\sigma)\right)
$$

where $p_{0}-p_{4}$ are the polynomial coefficients in Volts, Volts $* \mathrm{~cm}$, Volts $\mathrm{cm}^{2}$, Volts $* \mathrm{~cm}^{3}$, and Volts $* \mathrm{~cm}^{4}$ respectively. The units for these coefficients are assigned in the interest of keeping the output in terms of Volts, as seen by the photodetector. 
The fitting for the LI curve is carried out by code that is automatically generated by MATLAB's Curve Fitting Toolbox, which takes the initial guesses for the parameters and the expression to fit to as inputs. In this case the parameters are $\sigma_{0}, c$, and $\gamma$, where $\gamma$ is the pressure-broadened half width at halfmaximum. This automatically generated code is called in a main program to fit each spectral line separately. A fit of the spectral line at approximately $13103 \mathrm{~cm}^{-1}$ in the LI curve is shown in Fig. 10.

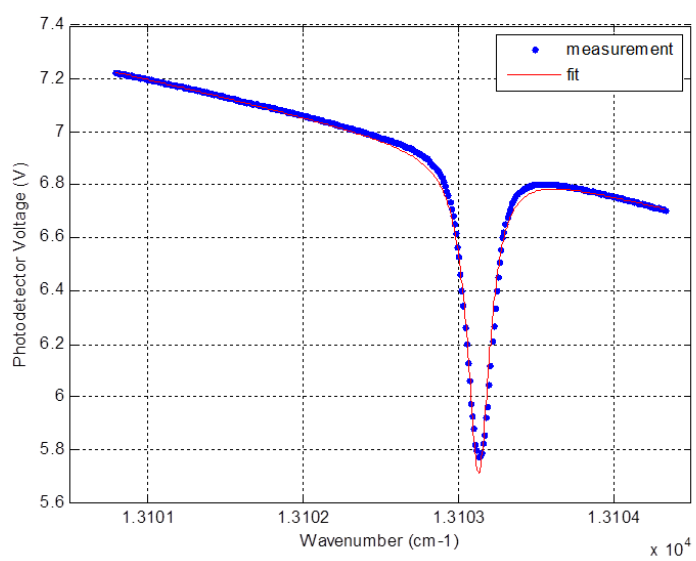

Fig. 10. LI curve fit

The fitted parameters from the fitting for the LI curve for each peak are shown in Table 1.

\begin{tabular}{|c|c|c|}
\hline$\frac{\left.\text { Concentration (molecules } / \mathrm{cm}^{3}\right)}{}$ & $\frac{\text { Fitted pressure-broadened }}{\text { HWHM }\left(\mathrm{cm}^{-1}\right)}$ & $\begin{array}{c}\text { HITRAN pressure- } \\
\text { broadened HWHM }\left(\mathrm{cm}^{-1}\right)\end{array}$ \\
\hline $2.2505 \mathrm{E}+19$ & 0.092820 & 0.048 \\
\hline $2.2320 \mathrm{E}+19$ & 0.090281 & 0.048 \\
\hline $2.2445 \mathrm{E}+19$ & 0.086262 & 0.049 \\
\hline $2.2802 \mathrm{E}+19$ & 0.087301 & 0.049 \\
\hline $2.2504 \mathrm{E}+19$ & 0.082221 & 0.051 \\
\hline $2.2795 \mathrm{E}+19$ & 0.081859 & 0.051 \\
\hline $2.2941 \mathrm{E}+19$ & 0.079327 & 0.053 \\
\hline $2.2565 \mathrm{E}+19$ & 0.076030 & 0.053 \\
\hline $2.3039 \mathrm{E}+19$ & 0.075105 & 0.055 \\
\hline $2.2647 \mathrm{E}+19$ & 0.072810 & 0.055 \\
\hline $2.2025 \mathrm{E}+19$ & 0.070000 & 0.058 \\
\hline
\end{tabular}

Table 1. Fitted Parameters for LI Curve

For WMS measurements, the current is modulated with a sinusoid as

$$
I(t)=I_{0}+m \sin \omega t
$$

where $I_{0}$ is a given drive current. As Fig. 7 illustrates, the relationship between the current and the wavenumber is not exactly linear, meaning that the wavenumber modulation does not have a sinusoidal profile. Rather, the wavenumber at a given time $t$ is a function of $I(t)$ :

$$
\sigma_{m}(t)=f(I(t))
$$

where $\sigma_{m}(t)$ is the modulated wavenumber at time $t$. This relationship is determined by carrying out a fourth degree polynomial fit of the current-wavenumber calibration data.

To extract the second harmonic at a specific drive current, or center frequency, the lockin amplifier multiplies the input from the photodetector with a $2 \omega$ sinusoid: 


$$
\left(p_{4} \sigma_{m}(t)^{4}+p_{3} \sigma_{m}(t)^{3}+p_{2} \sigma_{m}(t)^{2}+p_{1} \sigma_{m}(t)+p_{0}\right) \exp \left(-c d S g_{V}\left(\sigma_{m}(t)\right)\right) \cos 2 \omega t
$$

This signal is then passed through a lowpass filter to extract the DC component representing the second harmonic. In MATLAB, this involves extracting the first element of the array produced by taking the Fourier transform of the mixed signal using the function $\mathrm{fft}()$. The first element corresponds to the DC component of the mixed signal.

This process does not have a simplified form that can be used as an input to the Curve Fitting Toolbox, therefore it must be calculated numerically. The main program for the WMS data fitting code utilizes MATLAB function GlobalSearch supplied by the Global Optimization Toolbox. GlobalSearch utilizes another MATLAB function, fmincon, which finds the constrained minimum of a specified function. The program passes a cost function, which in

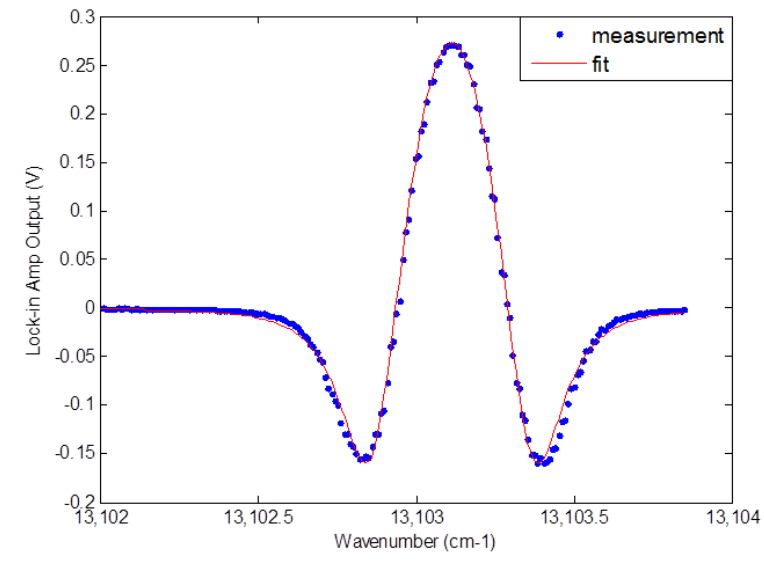

Fig. 11. WMS fit this case is defined as the sum of the squares of the difference between the measured and simulated data at each point, to GlobalSearch as an input, along with the parameters to be fitted. The WMS data consists of an additional parameter, the modulation amplitude $m$, which needs to be fitted. Although the modulation amplitude is set to a certain value during measurements, this parameter accounts for losses in signal strength at the VCSEL. The above numerical process is evaluated simply by sampling the value of the DC component of the mixed signal over one period. Fig. 11 shows the fitting of one of the spectral lines in the WMS data. Table 2 shows the fitted parameters and concentration for the fit of the spectral line shown in Fig. 11.

\begin{tabular}{|c|l|c|c|l|l|}
\hline $\begin{array}{l}\text { Expected } \\
\text { concentration } \\
\left(\mathrm{mol} / \mathrm{cm}^{3}\right)\end{array}$ & $\begin{array}{l}\text { Fitted } \\
\text { concentration } \\
\left(\mathrm{mol} / \mathrm{cm}^{3}\right)\end{array}$ & $\begin{array}{l}\text { HITRAN } \\
\text { HWHM }\left(\mathrm{cm}^{-1}\right)\end{array}$ & $\begin{array}{l}\text { Fitted HWHM } \\
\left(\mathrm{cm}^{-1}\right)\end{array}$ & $\begin{array}{l}\text { Applied } \\
\text { modulation } \\
\text { amplitude } \\
(\mathrm{mA})\end{array}$ & $\begin{array}{l}\text { Fitted } \\
\text { modulation } \\
\text { amplitude } \\
(\mathrm{mA})\end{array}$ \\
\hline $2.503 \mathrm{E}+19$ & $2.780 \mathrm{E}+19$ & 0.0510 & 0.1078 & 35 & 27.76 \\
\hline
\end{tabular}

Table 2. Fitted Parameters for WMS

The function fmincon may be used by itself, but it often tends to optimize towards the closest local minimum as opposed to the global minimum. GlobalSearch, on the other hand, generates a set of random starting points close to the specified starting point and within user defined bounds. The function then calls fmincon from each of the starting points, which greatly increases the probability of obtaining the global minimum. Despite this advantage the initial guess must still be relatively close to the global minimum. 
The expected concentration at $100 \% \mathrm{O}_{2}$ is calculated using the ideal gas law. The average fitted values of concentration for the LI curves deviate from the expected value by approximately $10 \%$ and the fitted value of concentration for the tested peak in the WMS data deviates from the expected value by approximately $11 \%$. The fitted pressure-broadened linewidth is approximately two times the value provided by HITRAN for the WMS data and approximately 1.2 to 2 times this value for the direct absorbance spectroscopy LI curves. This error may be due to additional broadening effects in the cell or a scalar constant in the experimental setup or in the numerical analysis of the data that is not accounted for. Further studies of the experimental setup, including the electronic equipment used for the experiment, as well as a reconfirmation of the validity of the data analysis will need to be carried out to find the source of this error.

\section{Conclusion}

Direct absorbance spectroscopy and wavelength modulation spectroscopy were carried out in a White cell with an optical path length of $2.6 \mathrm{~m}$. WMS was observed to be much more sensitive to low concentrations. So far, concentrations as low as $200 \mathrm{ppm}$ of $\mathrm{O}_{2}$ have been successfully detected. The concentration of $\mathrm{O}_{2}$ and the width of spectral lines is determined through fitting the data to a numerical expression for the WMS output. 


\section{References}

1. M. Bora et al., Proceedings SPIE 8366, 836607 (SPIE, Baltimore, MD, 2012).

2. J.M. Ostermann et al., IEEE Photon. Technol. Lett., 17, 11, 2256 (2005).

3. R. A. McCLatchey et al., Air Force Cambridge Research Laboratory Environmental Research Papers, AFCRL TR-73-0096 (AFCRL, Bedford, MA, 1973).

4. M. Lackner, Rev. Chem. Eng., 23, 2, 65 (2007).

5. J. U. White, J. Opt. Soc. Am., 32, 285 (1942).

6. Multi Reflection Gas Measurement Cells for Absorption Spectroscopy, http://www.ipm.fraunhofer.de/content/dam/ipm/de/PDFs/produktblaetter/AMS_Analysen _Messsysteme/SAW/Gasmesszelle_WEB.pdf, Jun. 2012.

7. E. E. Whiting, J. Quant. Spectrosc. Radiant. Transfer, 8, 1379 (1968). 